\title{
Research on Image Encryption Based on Lorenz Chaotic Mapping in Finite Field
}

\author{
Fangtao Liu ${ }^{1}$ and $\mathrm{Yu}$ Liu ${ }^{1,2}$ \\ ${ }^{1}$. Chongqing Vocational Institute of Engineering, Chongqing 402260, China \\ 2. State Key Laboratory of Coal Mine Disaster Dynamics and Control, Chongqing \\ University, Chongqing 400044, China \\ 8551756@qq.com
}

\begin{abstract}
An image encryption method was built based on Lorenz chaotic mapping and finite field theory. First, original image information was divided intryo image matrix by using Lorenz chaotic mapping. Second, five step calculations were finished about these two image matrix in the finite field. By combination of chaotic mapping and computing in the finite field, the new encryption method has a good effect and a fast speed. And the new encryption method realiz the balance of speed and effect. Experimental results show that the proposed method has good encryption effect, attack resistance, the ideal speed of execution.
\end{abstract}

Keywords: image encryption, finite field, Lorenz mapping, chaotic encryption

\section{Introduction}

With the rapid development of communication technology, the demand of large data file transmission is growing up. Among hem, image file transmission is a very common communication needs [1] the process of image data transmission, people have high demands on the security of image information, that promotes the rapid development of image encryption technology [2]. Image encryption is a method which can map original image information into cipher image information by using all kinds of encryption methods. Inage encryption can make the attacker and the translator not obtain the original image information. At the beginning of the image encryption technology development, stream cipher and matix scrambling were used more[3-4]. But these encryption methods are so simple than dectyption process is vulnerable to decode by attacker. This makes more complex encryption processing technology be introduced into image encryption field. Image encryption technology based on chaotic theory had been applied in recent years because chaotic mapping has a complex process and is sensitive to initial value. These factors make encryption safety greatly improved. Raiput used chaotic mapping to reschedule and layout the original image information, and built a new image encryption algorithm by using special treatment of initial sensitivity. This new encryption algorithm go a good encryption effect [5]. Chen set up a dynamic vector update mechanism to the process of image encryption based on chaotic mapping, in order to achieve the purpose which can improved the speed of image encryption and decryption[6]. Wen built a image encryption method that its security is very high. He combined Tent mapping with Hyper mapping, and further strengthen anti-attack performance of chaotic encryption. The safety performance of this method had been verified by $6^{\text {th }} \mathrm{CNN}$ attacking performance test [7]. Rohith used logistic to further dispose the key of encryption algorithm, and set up a linear transformation mechanism to further improve its security for image encryption algorithm based on chaotic theory [8]. 
Image encryption method based on chaotic theory has a strong advantage on the security, but its speed is generally slower. Therefore, a new image encryption method was built by combining finite field theory with chaotic theory.

\section{The Proposed Algorithm}

\subsection{Encryption Process Design}

The image encryption algorithm has built in this paper. It is combined the binary arithmetic on the finite field with chaotic encryption, and it can give full play to the advantages. That is, security and rapidity can be highlighted for image encryption at the same time. For this reason, the encryption process of the encryption method is presented as shown in Figure 1.

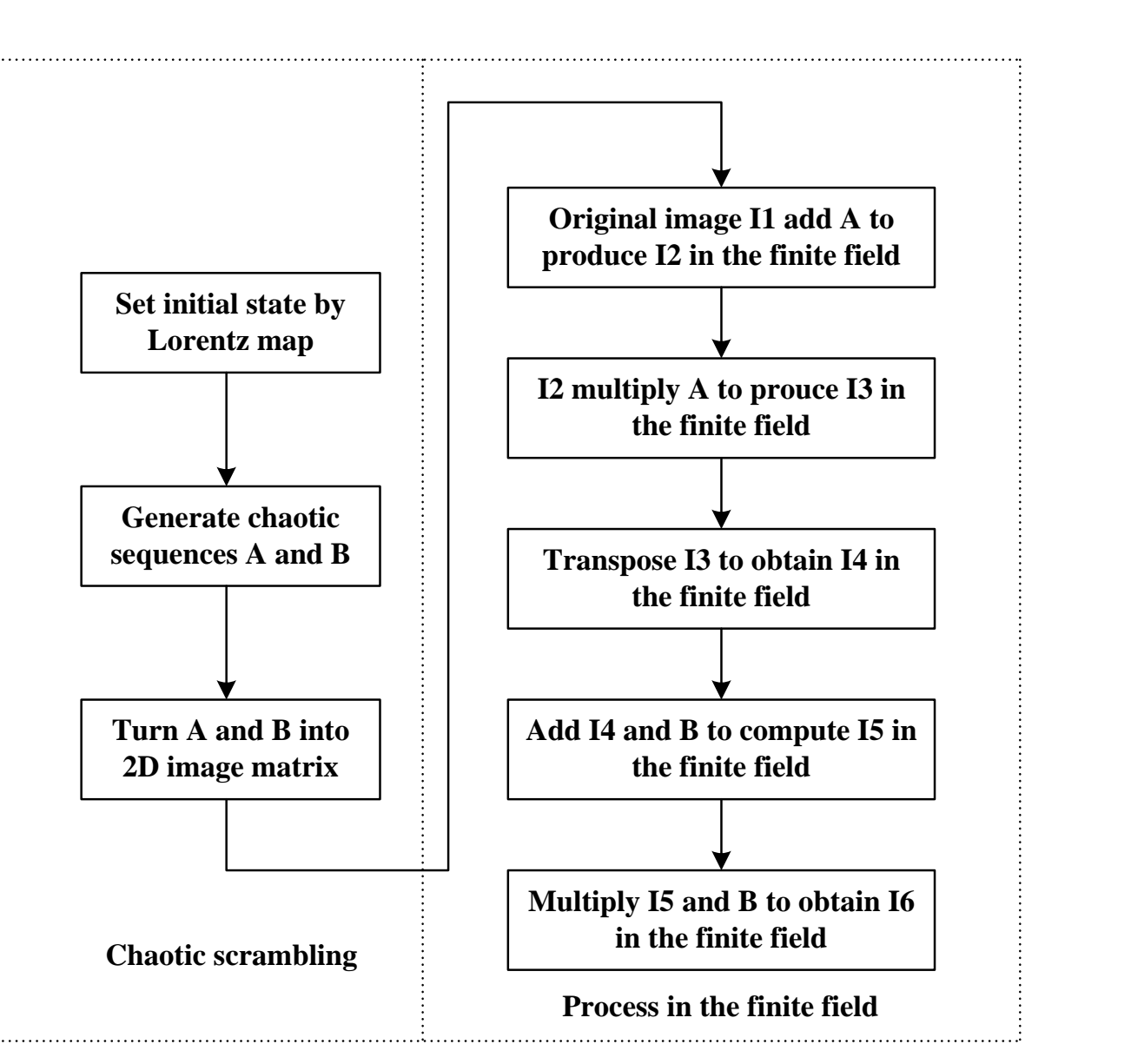

Figure 1. Encryption Principle and Encryption Process

\subsection{Chaotic Scrambling Process}

In the processing of the chaotic scrambling disposal, simple and effective chaotic mapping was used in order to accelerate the speed of encryption. Here, Lorenz chaotic mapping was used and it was shown in formula (1). 


$$
\left\{\begin{array}{l}
x_{1}=10\left(x_{2}-x_{1}\right) \\
x_{2}=28 x_{1}-x_{2}+x_{4}-x_{1} x_{3} \\
x_{3}=-\frac{8}{3} x_{3}+x_{1} x_{2} \\
x_{4}=-\frac{28}{3} x_{1}+x_{2}
\end{array}\right.
$$

Here, we put the original image information as $x_{1}$ into the formula (1), and generate other three variables $x_{2}, x_{3}, x_{4}$ by using Lorenz chaotic mapping.

We dispose $x_{3}$ and $x_{4}$ to make it just map between $0-255$, in order to satisfy the demand of numerical range of digital images. Specific treatment method is shown in formula (2).

$$
\left\{\begin{array}{l}
A=\left(x_{3} * 1000\right) \bmod 256 \\
B=\left(x_{4} * 1000\right) \bmod 256
\end{array}\right.
$$

By above process, $\mathrm{A}$ and $\mathrm{B}$ had become an one-dimensional séquence lata. In order to satisfy the requirement of the matrix operations on the finite field, $A$ and $B$ will map into image matrix, shown as in formula (3).

$$
\begin{aligned}
& A_{M \times N}=A_{n} \\
& B_{M \times N}=B
\end{aligned}
$$

\subsection{Finite Field Process}

Use I1 to express the originalimage, respectively execute additive operation and multiplication operation with A on the finite field. Then new image I 2 and I 3 will be obtained as follows:

$$
\text { 2) } \quad I 2=I 1 \oplus A
$$

Where, “ $\oplus$ "express addition onf finite field, “•” express multiplication on finite field.

Use transposition operation to obtain the new image I3, as shown in formula (6). Image 14 was respectively executed addition operation and multiplication operation with $\mathrm{B}$ on the finite field, new image I5 and I6 is obtained as follows.

$$
\begin{aligned}
& I 5=I 4 \oplus B \\
& I 6=I 5 \bullet B
\end{aligned}
$$

\subsection{Decryption Process Design}

On the receiver of encryption image transmission, you need to use the decryption alonithm to get the original image information. Here, receiver need to use the same Lorenz chaotic mapping function and the state of initial value with encryption end, and corresponding chaotic sequence to complete decryption. Rest of steps are equal to the inverse transformation of encryption process.

The first step, set the initial state according to the Lorentz mapping.

The second step, generate chaotic sequence A and B according to the Lorentz mapping and the original image information.

The third step, converts A and B to 2D image matrix.

The fourth step, make image I6 and B to execute the inverse operation of multiplication on finite field to get image I5. 
The fifth step, make image I5 and B to execute the inverse operation of addition on finite field to get image I4.

The sixth step, make the image I4 to execute transposition process to get image I3.

The seventh step, make image I3 and A to execute the inverse operation of multiplication on finite field to get image I2.

The eighth step, make image I 2 and A to execute the inverse operation of addition on finite field to get image I1.

\section{Experimental Results and Analysis}

In order to verify the effectiveness of image encryption method based on fusion of chaotic scrambling and finite field, test and analysis is executed on encryption effect. Barbara image and Bahoon image is selected as experimental image, experimental results are shown in Figure 2 and Figure 3.

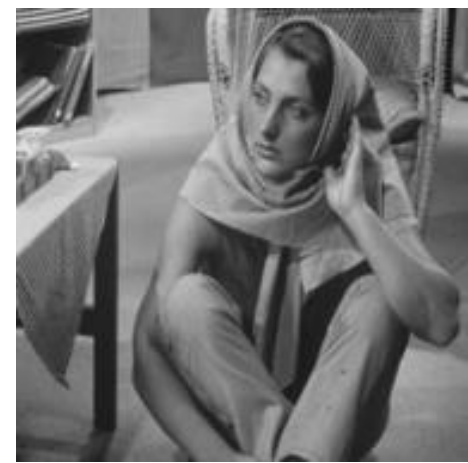

a) original image

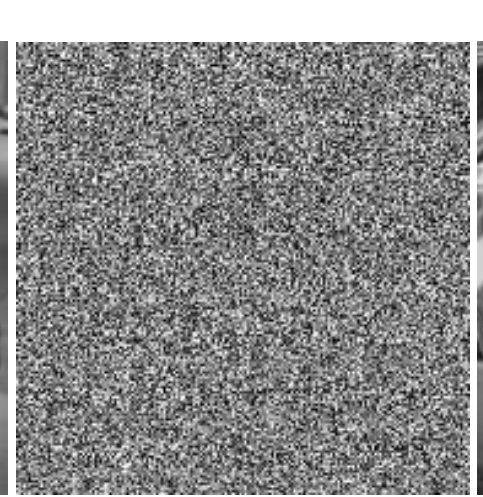

b) encryption image

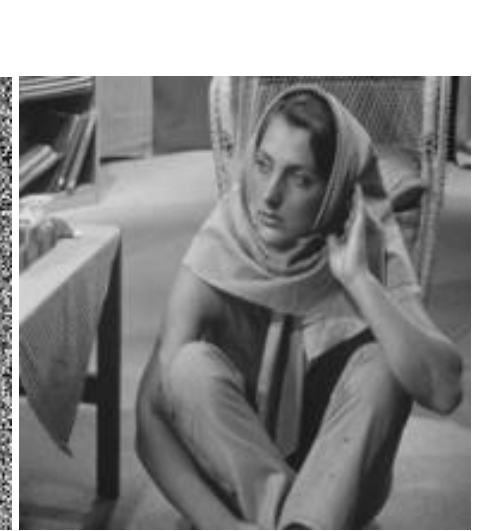

c) decryption image

Figure 2. Ehcryption Effect of Barbara Image

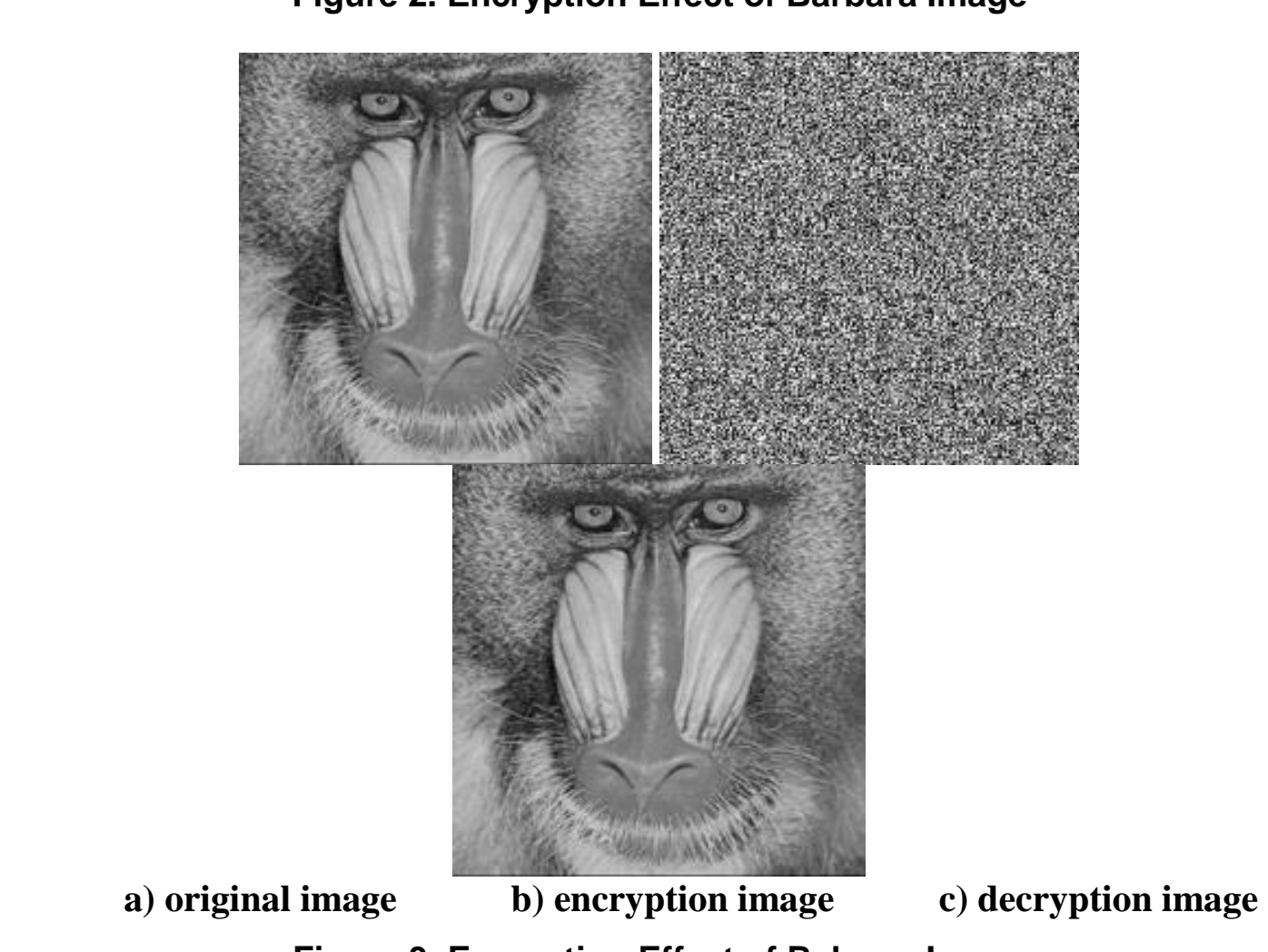

Figure 3. Encryption Effect of Bahoon Image 


\subsection{Gray Histogram Evaluation}

Observe gray histogram original image and encryption image, it is also an effective safety evaluation method. In general, image texture feature is more obvious, the gray scale distribution is more concentrated. After encryption process, the distribution of gray histogram is more homogeneous. The better Homogeneity is, the better encryption effect is. Histogram effect of Barbara original image and encryption image is shown as Figure 4.

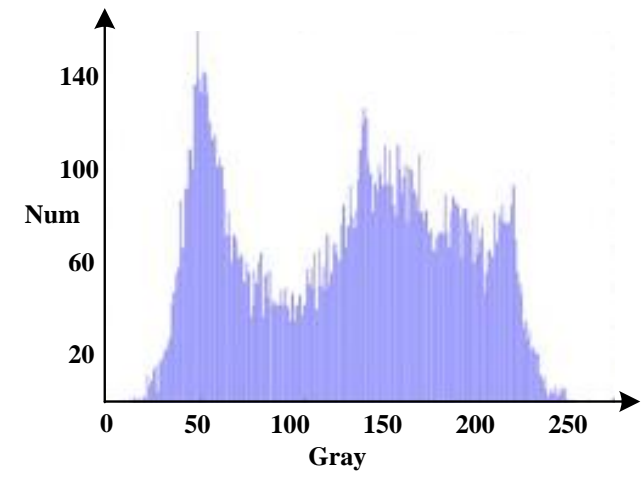

a) Original image

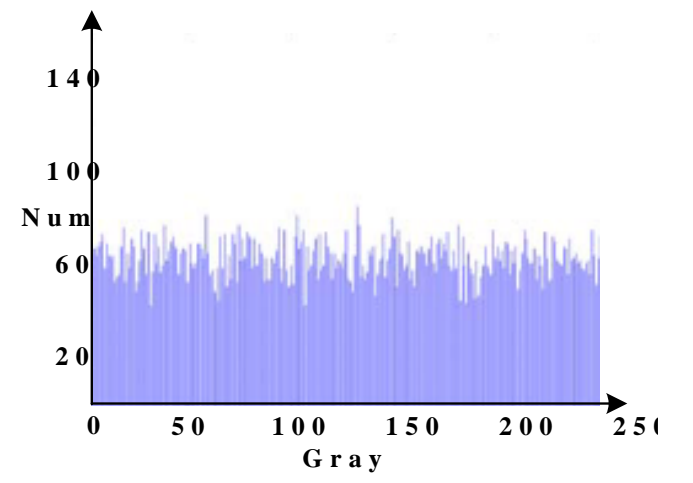

b) Encryption image

Figure 4. Gray Histogram Evaluation

From Figure.4, we can see that gray histogram distribution of encryption image is getting very uniform. So it can prove that encryption security of the proposed method is higher.

\subsection{Three-Way Correlation Evaluation}

Three-way correlation refers to the correlation of image pixels among the horizontal direction, vertical direction, and diagonal direction. The correlation no matter which direction is high, it will make image date easily to decode. Therefore, to measure encryption performance is good or not the most direct way is evaluating the correlation of these three directions. When the correlation is calculated, statistical formula are generally used as follows.

$$
\begin{aligned}
& E(x)=\frac{1}{N} \sum_{i=1}^{N} x_{i} \\
& D(x)=\frac{1}{N} \sum_{i=1}^{N}\left(x_{i}-E(x)\right)^{2} \\
& \operatorname{cov}(x, y)=\frac{1}{N} \sum_{i=1}^{N}\left(x_{i}-E(x)\right)\left(y_{i}-E(y)\right) \\
& r_{x y}=\frac{\operatorname{cov}(x, y)}{\sqrt{D(x)} \times \sqrt{D(y)}}
\end{aligned}
$$

By using above formula, three directions correlation coefficient are calculated on Barbara image, and results are shown in Table 1 
Table 1. Correlation Results

\begin{tabular}{ccc}
\hline correlation coefficient & Barbara original image & Barbara encryption image \\
\hline $\begin{array}{c}\text { The correlation coefficient of } \\
\text { horizontal direction }\end{array}$ & 0.9250 & 0.0021 \\
$\begin{array}{c}\text { The correlation coefficient of } \\
\text { vertical direction }\end{array}$ & 0.9550 & 0.0016 \\
$\begin{array}{c}\text { The correlation coefficient of } \\
\text { diagonal direction }\end{array}$ & 0.8890 & 0.0017 \\
\hline
\end{tabular}

From Table 1, we can see that correlation coefficient of three directions are very low. The results show that the proposed method has a high safety.

\subsection{Execution Time Analysis}

For Barbara and Bahoon images, the execution time of the proposed method are shown as in Table 2.

Table 2. Execution Time

\begin{tabular}{ccc}
\hline Image size & Encryption time (second) & Decryption time(second) \\
\hline Barbara image & 0.084 & 0.079 \\
Bahoon image & 0.092 & 0.085 \\
\hline
\end{tabular}

\section{Conclusions}

In order to get better image encryption effect and encryption speed, a new image encryption was built based on the finitefield and Loren chaotic mapping. In this method, original image information was mapped into two matrices, and final encryption result can be obtained by all kinds of operation on the finite field. In the test experiment, we analyzed the encryption effect, histogramevalation effect, three-way correlation effect, and execution time of this method. The experimental results show that the encryption effect is good, anti-attack/performance is strong, and the execution time is fast. The proposed method gives full play to the characteristics which the chaotic encryption effect is good and the operation speed is fast on finite field.

\section{References}

[1] D. Maluenda, A. Carnicer, R. Martnez-Herrero, I. Juvells and B. Javidi, "Optical encryption using photon-counting polarmetric imaging", Optics Express, vol. 23, (2015), pp. 655-666.

[2] N.F. Elabady, H.M. Abdalkader, M.I. Moussa and S.F. Sabbeh, "Image encryption based on new onedimensional chaotic map", ICET 2014-2 ${ }^{\text {nd }}$ International Conference on Engineering and Technology, (2015), pp. 851-892.

[3] H. Singh, A.K. Yadav, S. Vashisth and K. Singh, "Double phase-image encryption using gyrator transforms, and structured phase mask in the frequency plane", Optics and Lasers in Engineering, vol. 67 (2015), pp.145-156.

[4] Morales, L. Daz and C. Torres Radia, "Hilbert transform in terms of the fourier transform applied to mage encryption", Journal of Physics Conference Series, vol. 1, (2015), pp. 582-590.

[51 R. A. Singh and S. Mansi, "A novel image encryption and authentication scheme using chaotic maps", Advances in Intellgent Systems and Computing, (2015), pp. 277-286

[6] J.X. Chen, Z.L. Zhu, C. Fu and H. Yu, "A fast chaos-based image encryption scheme with a dynamic state variables selection mechanism", Communications in Nonlinear Science and Numerical Simulation, vol. 20, (2015), pp.846-860.

[7] W. Wen, "Security analysis of a color image encryption scheme based on skew tent map and hyper chaotic system of 6-order CNN against chosen plaintext attack", Multimedia Tools and Applications, 2015, vol. 6, (2015), pp.224-235.

[8] S. Rohith, K.N. Bhat, S. Hari and A. Nandini, "Image encryption and decryption using chaotic key sequence generate by sequence of logistic map and sequence of states of Linear Feedback Shift Register", International Conference on Advances in Electronic, Computers and Communications, vol. 6 , (2015), pp.1124-1130. 


\section{Authors}

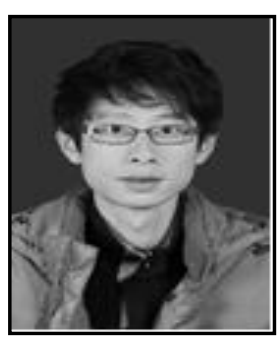

Fangtao Liu, he is an Engineer , School of Information Engineering, Chongqing Vocational Institute of Engineering, Chongqing 402260, China;Tel: +86-023-61065852;:Email: 8551756@qq.com

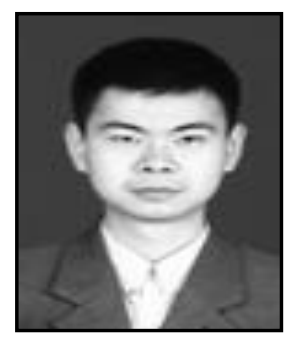

Yu Liu, he is a Professor, School of Information Engineering,Chongqing Vocational Institute of Engineering, Chongqing 402260, China; Tel: +86-023-65208186; Email:16619054@qq.com

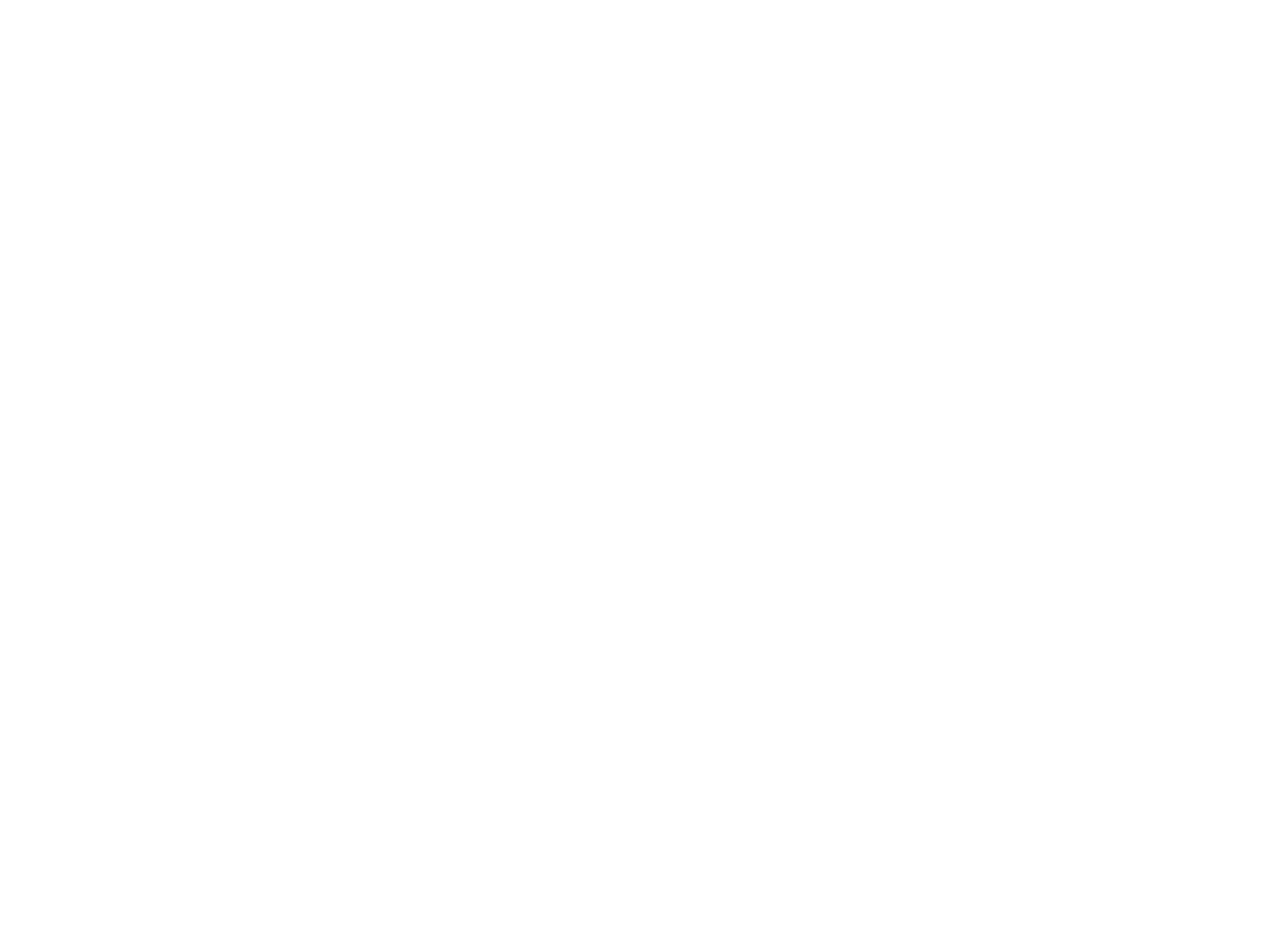


International Journal of Hybrid Information Technology

Vol. 9, No.10 (2016)

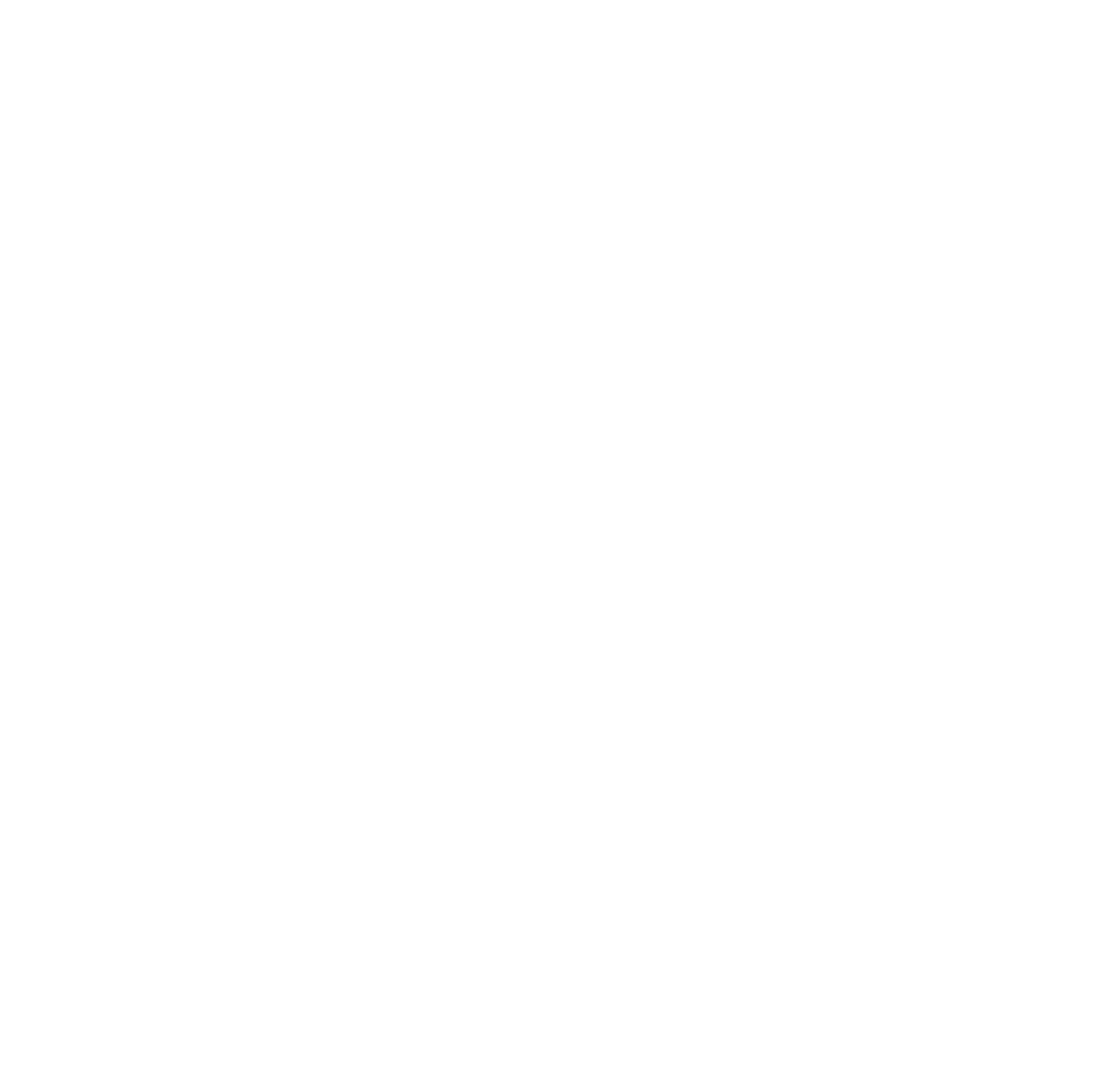

